\title{
Inter- and intra-observer reliability of masking in plantar pressure measurement analysis
}

\author{
K. Deschamps ${ }^{\text {a,c,* }}$, I. Birch ${ }^{\text {b }}$ J. Mc Innes ${ }^{\text {b }}$, K. Desloovere ${ }^{c, d}$, G.A. Matricali ${ }^{\text {a,c }}$ \\ a Division of Musculoskeletal Disorders, University Hospitals Leuven, KU Leuven, Leuven, Belgium \\ ${ }^{\mathrm{b}}$ Podiatry Division, School of Health Professions, University of Brighton, Eastbourne, United Kingdom \\ ' Clinical Motion Analysis Laboratory, University Hospitals Leuven, KU Leuven, Leuven, Belgium \\ ${ }^{\mathrm{d}}$ Department of Rehabilitation Sciences, Katholieke Universiteit Leuven, Leuven, Belgium
}

\section{A R T I C L E I N F O}

\section{Article history:}

Received 13 January 2009

Received in revised form 16 June 2009

Accepted 26 June 2009

\section{Keywords:}

Plantar pressure

Standardization

Reliability

Masking

Inter-observer

Intra-observer

\begin{abstract}
A B S T R A C T
Plantar pressure measurement is an important tool in gait analysis. Manual placement of small masks (masking) is increasingly used to calculate plantar pressure characteristics. Little is known concerning the reliability of manual masking. The aim of this study was to determine the reliability of masking on $2 \mathrm{D}$ plantar pressure footprints, in a population with forefoot deformity (i.e. hallux valgus). Using a random repeated-measure design, four observers identified the third metatarsal head on a peak-pressure barefoot footprint, using a small mask. Subsequently, the location of all five metatarsal heads was identified, using the same size of masks and the same protocol. The 2D positional variation of the masks and the peak pressure (PP) and pressure time integral (PTI) values of each mask were calculated. For single-masking the lowest inter-observer reliability was found for the distal-proximal direction, causing a clear, adverse impact on the reliability of the pressure characteristics (PP and PTI). In the medial-lateral direction the inter-observer reliability could be scored as high. Intra-observer reliability was better and could be scored as high or good for both directions, with a correlated improved reliability of the pressure characteristics. Reliability of multi-masking showed a similar pattern, but overall values tended to be lower. Therefore, small sized masking in order to define pressure characteristics in the forefoot should be done with care. (C) 2009 Elsevier B.V. All rights reserved.
\end{abstract}

\section{Introduction}

Plantar pressure measurement equipment is used in many areas of health science [1-5]. This equipment is now considered to be an invaluable tool in the assessment of 'feet at risk' [6] and in integrated biomechanical approaches combining EMG, 3D motion analysis and force plate $[7,8]$.

When performing plantar pressure measurements and analysis, the investigator is faced with two types of challenges that may strongly affect the validity and reliability of this technique. The first is related to the technical characteristics of the equipment used [912]. The second is caused by problems associated with the accurate identification of anatomical structures on pressure-based footprints.

In order to facilitate and standardize pressure analysis, the footprint is frequently divided into regions of interest (ROI), for which physical quantities such as peak pressure values and impulses can be determined. A wide range of definitions of such

\footnotetext{
* Corresponding author at: Division of Musculoskeletal Disorders, U.Z. Leuven, KU Leuven, Weligerveld 1, B-3212 Pellenberg (Lubbeek), Belgium. Tel.: +32 16338827; fax: +32 16338824 .

E-mail address: kevin.deschamps@uz.kuleuven.ac.be (K. Deschamps).
}

ROI have been proposed and used in the past. A number of authors have relied on ROI automatically defined by the software of the pressure measurement system used [13-15]. The major justification for this approach, based upon geometric algorithms, is the standardization of the analysis $[16,17]$. Others have used manual definitions for ROI determination, frequently called 'masks' [1821]. These masks represent a rectangular area of $1.5-3.5 \mathrm{~cm}^{2}$ and are intended to identify an area of a limited number of sensors. This method allows a high degree of flexibility that may be important when evaluating different types of foot deformities. Unfortunately, an increased flexibility has the potential risk of being unrepeatable and therefore not suitable for scientific purposes.

The aim of this study was to evaluate the reliability of manually determined masks in plantar pressure footprints. The study hypothesis was that manual masking on plantar pressure measurements of patients with first ray deformity is not a reliable method to evaluate plantar pressure characteristics.

\section{Methods \\ 2.1. Subjects}

The pre-operative plantar pressure measurements of all patients $(n=56)$ who underwent hallux valgus surgery during a 6-month period at our Foot \& Ankle Department were reviewed for the purpose of this investigation. Participants were 
excluded if they presented with neurological disorders, amputations, diabetes, systemic inflammatory diseases and history of previous foot surgery. Eleven patients were excluded based on these criteria, and a further nine patients were excluded due to inadequate data (incomplete files). From the remaining 36 patients, 10 were selected using a random selection table to participate in this investigation. The final cohort was composed out of nine women and one man and had a mean age of 56 years. The mean hallux valgus angle was $33^{\circ}$ and the mean first intermetatarsal angle was $13.5^{\circ}$, based on X-ray measurements using the Picture Archiving and Communication System of the University Hospital. The study protocol was approved by the Institution's Review Board.

\subsection{Plantar pressure equipment}

The plantar pressure measurements were performed using a pressure platform (RS Scan International, Olen, Belgium). The dimensions of the platform were $50 \mathrm{~cm} \times 40 \mathrm{~cm} \times 0.8 \mathrm{~cm}$, consisting of 4096 force sensing resistors, each $0.75 \mathrm{~cm} \times 0.5 \mathrm{~cm}$ in size, organized in 64 rows and 64 columns. The platform was mounted level with the surrounding floor. Gait analysis was performed according the guidelines described by Harrison and Folland [22] and the Foot Pressure Interest Group. Pressure data were analysed using the RS Scan software version 7.0 .

\subsection{Study design}

A random repeated-measure design was used to allow inter- and intra-observer reliability testing. Four observers, two podiatry students and two experienced podiatrists participated in this study. From each subject $(n=10)$, one peak-pressure footprint was used for further analysis. In the first part $(A)$ of the study, the four observers identified the third metatarsal head (MTH) region (single-masking) on the peak-pressure footprint, using one mask (size: $1.5 \mathrm{~cm} \times 1.0 \mathrm{~cm}$ ). Ten repeated measurements were made by each of the 4 observers on the peak-pressure footprints (a total of 400 measurements) on five occasions during a 2-week time span, minimizing the possibility of the observers memorizing the mask positions. Subsequently, 2 weeks later, the multi-masking procedure (part B) for each of the $5 \mathrm{MTH}$ was performed on the same footprints using the same study design (a total of 2000 measurements). During masking the dorso-plantar plain X-ray of the patient's foot was available to the observer in order to visualize the individual foot morphology as none of the observers saw the subjects' feet clinically. Each observer was given time to become familiar with the masking procedure before initiating the study.

The absolute peak pressure (PP) and pressure time integral (PTI) values of each mask were calculated. Both parameters were considered because previous studies have found a strong correlation between these parameters and a number of pathological conditions $[2,14,15]$, and because they are calculated in a different way. The PP determined by the software represents the highest pressure recorded by one of the four sensors of the mask. For the PTI the software will take into account the pressure recording of the four sensors and this with respect to time.

To further explore the inter-observer agreement for the multi-masking procedure, ensemble averages of the peak-pressure values for all metatarsal heads were calculated out of the ten assessments made for every footprint by each observer.

\subsection{Cartesian coordinates}

To track the changes in mask position, the 2D footprints were analysed using Corel Draw $12^{\mathrm{TM}}$ (Corel, Dublin, Ireland). After masking, the peak-pressure footprint image was imported into Corel Draw $12^{\mathrm{TM}}$ by using Corel CAPTURE ${ }^{\mathrm{TM}}$ (Corel). Using the basic Cartesian reference frame of Corel Draw $12^{\mathrm{TM}}$, calibration of the image was done based upon the known size of the masks. Such a scaling factor is also used by the manufacturer in order to visualize the foot print adequately in the software. The same basic Cartesian reference frame was further used to determine the $x-y$ coordinates of the centre of each mask. Using the graphical tools of Corel Draw $12^{\mathrm{TM}}$, the margins of each mask were reconstructed. Subsequently the centre of the rectangular mask was determined by drawing the diagonals of the mask. The $x$ and $y$ coordinates of the intersecting point of both diagonals were considered as to be centre of the mask. The $x$-axis of the coordinate frame corresponded to the mediolateral axis of the foot, the $y$-axis to the distal-proximal axis. All measurements were made with a precision of $0.1 \mathrm{~mm}$. Representing each rectangular mask by only one $x$ and $y$ coordinate had two major advantages. It simplified the data analysis and allowed the positional changes of one point in the fixed reference system to be unambiguously calculated.

Preliminary, the repeatability of determining the $x-y$ coordinates for this design was evaluated by one clinician, using a total of 20 plantar pressure measurements of five different subjects. In each footprint, the multi-masking procedure was performed. Subsequently, the centre of each mask was determined three times within a random selected order according to the above-described procedure.

\subsection{Statistics}

To quantify the repeatability of the $x$ and $y$ coordinates assessed in the preliminary study, the coefficient of variation (CV) was calculated.
ANOVA based intraclass correlation (ICC) techniques were used to estimate the inter- and intra-observer reliability. The following boundary conditions were used with respect to the ICC: $\geq 0.90$ high, $0.89-0.80$ good, $0.79-0.70$ fair, $\leq 0.69$ poor reliability [23]. The $95 \%$ lower confidence limit (LCL) was also calculated in order to estimate the uncertainty of the ICCs. The standard error of mean (SEM) was calculated as a direct measure of measurement accuracy. All statistical analyses were performed by S.A.S., 8.02 TS (SAS Institute Inc., Cary, NC). Absolute pressure values, the minimum and maximum PP and PTI values, found during the multi-masking procedure, were considered in order to allow a clinical interpretation of the results.

The coefficient of variation (mean CV) was calculated for each MTH in order to quantify the inter-observer agreement if the ensemble average is calculated out of ten measurements and this for each patient.

\section{Results}

The calibration method showed a CV of $1.09 \%$ for the length of the mask and $0.89 \%$ for the width. The repeatability of defining the $x-y$ coordinates of the middle point of the mask showed a CV of $0.22 \%$ for the $x$ coordinate and $0.1 \%$ for the $y$ coordinate.

The results of single-masking (Part A) are shown in Tables 1 and 2. In single-masking (Table 1), the observers achieved a high agreement for the $x$ coordinates (ICC $\geq 0.90$ ), and a good agreement for the $y$ coordinates (ICC $=0.86$ ). Considering the $95 \% \mathrm{LCL}$, the reliability coefficient did exceed the 0.8 level for the $x$ coordinates but not for the $y$ coordinates (fair $=0.73$ ). The interobserver reliability of the related pressure characteristics was only poor for the PTI and fair for the PP values (ICC $=0.79$ ). The LCL of both pressure characteristics decreased below the 0.69 level, and the SEM for the peak-pressure values was almost $35 \mathrm{kPa}$ and for the impulse $13 \mathrm{~s} / \mathrm{kPa}$. Considering the intra-observer reliability, the ICC scores were high for observer 1 (experienced) and 2 (non-

Table 1

Inter-observer Intraclass Correlation Coefficients (ICCs), standard error of mean (SEM) and 95\% lower confidence limit (LCL) for single-masking (third metatarsal head).

\begin{tabular}{lllllc}
\hline & \multicolumn{2}{l}{ Coordinates } & & \multicolumn{2}{l}{ Pressure characteristics } \\
\cline { 2 - 3 } \cline { 5 - 6 } & $X$-axis & Y-axis & & Peak pressure & Pressure time integral \\
\hline ICC & 0.91 & 0.86 & & 0.79 & 0.68 \\
SEM $^{\text {a }}$ & 3.73 & 4.02 & & 34.8 & 13.2 \\
LCL $^{n}$ & 0.83 & 0.73 & & 0.59 & 0.4 \\
\hline
\end{tabular}

a Note: the unit of the SEMs of the coordinates are millimetres ( $\mathrm{mm})$, for the peak pressure $(\mathrm{kPa})$ and for the pressure time integral $(\mathrm{s} / \mathrm{kPa})$.

Table 2

Intra-observer Intraclass correlation coefficients (ICCs), standard error of mean (SEMs) and 95\% lower confidence limit (LCL) for single-masking (third metatarsal head).

\begin{tabular}{|c|c|c|c|c|}
\hline & \multicolumn{2}{|c|}{ Coordinates } & \multicolumn{2}{|c|}{ Pressure characteristics } \\
\hline & $X$-axis & $Y$-axis & Peak pressure & Pressure time integral \\
\hline \multicolumn{5}{|c|}{ Observer 1} \\
\hline ICC & 0.99 & 0.97 & 0.96 & 0.96 \\
\hline $\mathrm{SEM}^{\mathrm{a}}$ & 1.32 & 1.34 & 14.7 & 4.2 \\
\hline $\mathrm{LCL}$ & 0.98 & 0.94 & 0.93 & 0.92 \\
\hline \multicolumn{5}{|c|}{ Observer 2} \\
\hline ICC & 0.95 & 0.9 & 0.93 & 0.91 \\
\hline SEM $^{\mathrm{a}}$ & 3.2 & 3.3 & 19.3 & 6.8 \\
\hline LCL & 0.89 & 0.8 & 0.87 & 0.82 \\
\hline \multicolumn{5}{|c|}{ Observer 3} \\
\hline ICC & 0.88 & 0.86 & 0.84 & 0.81 \\
\hline SEM $^{\mathrm{a}}$ & 3.78 & 3.09 & 29.6 & 9.4 \\
\hline $\mathrm{LCL}$ & 0.78 & 0.73 & 0.7 & 0.64 \\
\hline \multicolumn{5}{|c|}{ Observer 4} \\
\hline ICC & 0.86 & 0.91 & 0.86 & 0.9 \\
\hline SEM $^{\mathrm{a}}$ & 3.23 & 3.33 & 28.9 & 8.1 \\
\hline LCL & 0.74 & 0.8 & 0.74 & 0.88 \\
\hline
\end{tabular}

a Note: the unit of the SEMs of the coordinates are millimetres ( $\mathrm{mm})$, for the peak pressure $(\mathrm{kPa})$ and for the pressure time integral $(\mathrm{s} / \mathrm{kPa})$. 
Table 3

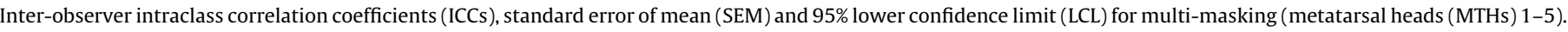

\begin{tabular}{|c|c|c|c|c|c|c|c|c|c|c|c|c|}
\hline & \multicolumn{6}{|c|}{ Coordinates } & \multicolumn{6}{|c|}{ Pressure characteristics } \\
\hline & \multicolumn{3}{|c|}{$X$-axis } & \multicolumn{3}{|c|}{$Y$-axis } & \multicolumn{3}{|c|}{ Peak pressure } & \multicolumn{3}{|c|}{ Pressure time integral } \\
\hline & ICC & SEM $^{\mathrm{a}}$ & $\mathrm{LCL}$ & ICC & SEM $^{\mathrm{a}}$ & $\mathrm{LCL}$ & ICC & SEM $^{\mathrm{a}}$ & LCL & ICC & SEM $^{\mathrm{a}}$ & $\mathrm{LCL}$ \\
\hline MTH 1 & 0.97 & 2.03 & 0.96 & 0.87 & 2.3 & 0.73 & 0.94 & 23.2 & 0.88 & 0.84 & 8.3 & 0.69 \\
\hline MTH 2 & 0.85 & 1.77 & 0.7 & 0.85 & 2.09 & 0.69 & 0.77 & 26.4 & 0.66 & 0.82 & 8.9 & 0.65 \\
\hline MTH 3 & 0.95 & 2.2 & 0.9 & 0.82 & 4.5 & 0.65 & 0.91 & 15.3 & 0.83 & 0.9 & 5.3 & 0.8 \\
\hline MTH 4 & 0.98 & 1.6 & 0.97 & 0.77 & 5.4 & 0.55 & 0.83 & 18.4 & 0.67 & 0.75 & 7.2 & 0.53 \\
\hline MTH 5 & 0.98 & 2.1 & 0.96 & 0.8 & 3.5 & 0.65 & 0.57 & 14. & 0.24 & 0.74 & 5.2 & 0.55 \\
\hline
\end{tabular}

a Note: the unit of the SEMs of the coordinates are millimetres $(\mathrm{mm})$, for the peak pressure $(\mathrm{kPa})$ and for the pressure time integral (s/kPa).

experienced) and good for observer 3 (experienced) and 4 (nonexperienced). Similar results were observed for the PP and PTI values, with the lowest reliability scores for observer 3.

Results of multi-masking (Part B) are shown in Tables 3 and 4. The results of the inter-observer ICC calculations (Table 3 ) all exceeded the 0.9 level for the $x$ coordinates, with exception of the second metatarsal $($ ICC $=0.85)$. The ICC scores for the $y$ coordinates were all good, with exception of the fourth MTH (ICC $=0.77)$. The related LCL scores ranged from fair to poor. Lower reliability values were found for almost all the pressure characteristics. The ICC scores of PP values of the fifth and second MTH showed poor reliability, for the PTI values it was the fourth and fifth MTH who demonstrated the lowest ICC scores (ICC $<0.79$ ). Table 4 demonstrates that good and high intra-observer reliability was achieved for the $x$ and $y$ coordinates, as well as for the pressure characteristics. Observers 1 and 4 showed the best intra-observer multi-masking repeatability. The minimum and maximum PP and PTI values found during the multi-masking procedure for the four observers was: for the first MTH (PP: 12-415 kPa, PTI 0-161 s/kPa), for the second MTH (PP: 111-403 kPa, PTI 32-130 s/kPa), the third MTH (PP: 152-442 kPa, PTI 58-131 s/kPa), the fourth MTH (PP: 64-382 kPa, PTI: 29-130 s/kPa) and for the fifth MTH (PP: 25$415 \mathrm{kPa}$, PTI: 5-103 s/kPa).

Fig. 1 shows the inter-observer agreement of the ensemble averaged PP for the second MTH, for each patient and each observer.

Table 4

Intra-observer 95\% lower confidence limit for multi-masking (metatarsal heads (MTHs) 1-5).

\begin{tabular}{|c|c|c|c|c|}
\hline & Observer 1 & Observer 2 & Observer 3 & Observer 4 \\
\hline \multicolumn{5}{|l|}{$X$-axis } \\
\hline MTH 1 & $\mathrm{X}$ & $\mathrm{X}$ & $\mathrm{X}$ & $\mathrm{X}$ \\
\hline MTH 2 & $\mathrm{X}$ & 0.77 & $\mathrm{X}$ & $\mathrm{X}$ \\
\hline MTH 3 & $\mathrm{X}$ & 0.65 & 0.71 & $\mathrm{X}$ \\
\hline MTH 4 & $\mathrm{X}$ & $\mathrm{X}$ & $\mathrm{X}$ & $\mathrm{X}$ \\
\hline MTH 5 & $\mathrm{X}$ & $\mathrm{X}$ & $\mathrm{X}$ & $\mathrm{X}$ \\
\hline \multicolumn{5}{|l|}{$Y$-axis } \\
\hline MTH 1 & $X$ & $\mathrm{X}$ & $\mathrm{X}$ & $\mathrm{X}$ \\
\hline MTH 2 & $\mathrm{X}$ & $\mathrm{X}$ & $\mathrm{X}$ & $\mathrm{X}$ \\
\hline MTH 3 & $X$ & $\mathrm{X}$ & $\mathrm{X}$ & $\mathrm{X}$ \\
\hline MTH 4 & $\mathrm{X}$ & 0.79 & $\mathrm{X}$ & $\mathrm{X}$ \\
\hline MTH 5 & $\mathrm{X}$ & $\mathrm{X}$ & $\mathrm{X}$ & $\mathrm{X}$ \\
\hline \multicolumn{5}{|c|}{ Peak pressure } \\
\hline MTH 1 & $\mathrm{X}$ & $\mathrm{X}$ & $\mathrm{X}$ & $\mathrm{X}$ \\
\hline MTH 2 & 0.57 & 0.59 & 0.55 & 0.70 \\
\hline MTH 3 & $\mathrm{X}$ & $\mathrm{X}$ & $\mathrm{X}$ & $\mathrm{X}$ \\
\hline MTH 4 & $\mathrm{X}$ & $\mathrm{X}$ & $\mathrm{X}$ & $\mathrm{X}$ \\
\hline MTH 5 & $X$ & $\mathrm{X}$ & $X$ & $\mathrm{X}$ \\
\hline \multicolumn{5}{|c|}{ Pressure time integral } \\
\hline MTH 1 & $\mathrm{x}$ & $\mathrm{X}$ & $\mathrm{X}$ & $\mathrm{X}$ \\
\hline MTH 2 & 0.61 & 0.53 & 0.58 & $\mathrm{X}$ \\
\hline MTH 3 & $\mathrm{X}$ & $\mathrm{X}$ & $\mathrm{X}$ & 0.66 \\
\hline MTH 4 & $\mathrm{X}$ & $\mathrm{X}$ & 0.74 & $\mathrm{X}$ \\
\hline MTH 5 & $\mathrm{X}$ & $\mathrm{X}$ & $\mathrm{X}$ & $\mathrm{X}$ \\
\hline
\end{tabular}

$X=95 \%$ lower confidence limit score $\geq 0.8$.

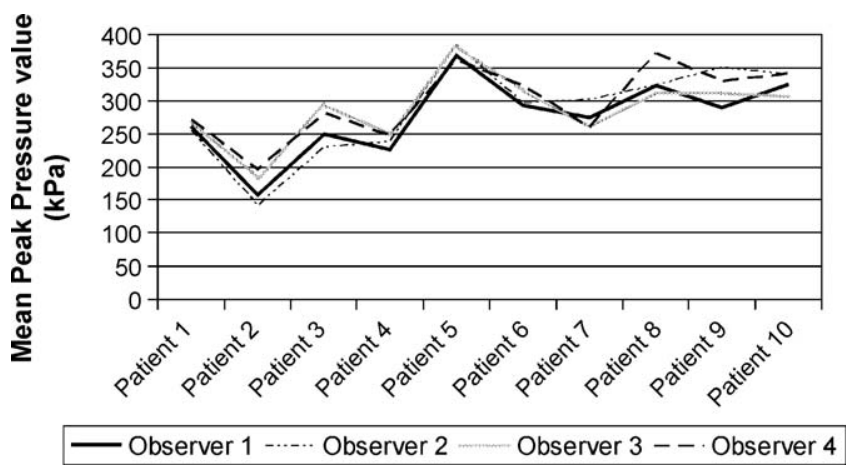

Fig. 1. Mean peak pressure at the second metatarsal head for ten assessments of one footprint calculated for every single patient. Comparison between the four observers shows an acceptable gross level of agreement for the inter-observer reliability.

The CV for the second MTH was 6.2\%. The mean CV for the first, third, fourth and fifth MTH was respectively 9.7\%, 5.5\%, 7.9\% and 7.9\%.

\section{Discussion}

The major objective of this study was to investigate the interand intra-observer reliability of single-masking (third MTH) and multi-masking (all $5 \mathrm{MTH}$ ) of $1.5 \mathrm{~cm} \times 1 \mathrm{~cm}(2 \times 2$ sensors) masks in a population with forefoot deformity (i.e. hallux valgus). The secondary objective was to estimate the effect of masking upon commonly used pressure characteristics such as peak pressure and pressure time integral.

Single-masking showed a high inter-observer reliability considering the medio-lateral direction and a good reliability in the proximal-distal direction (ICC $=0.86$ ). For the $L C L$, in the proximaldistal direction only fair reliability was obtained. Considering the pressure characteristics, ICC values for the PP and PTI were respectively only 0.79 and 0.68 . LCL values resulted inferior to 0.59 , which implies that inter-observer reliability is poor. Strikingly, when applying the aforementioned boundary conditions, singlemasking could be classified as good, but the clinical impact, represented by the PP and PTI, showed only a poor reliability.

The results of multi-masking showed again the highest interobserver reliability (ICCs between 0.85 and 0.98 ) in the mediallateral direction and a lower in the distal-proximal direction. The LCL scores of the $y$ coordinates ranged between 0.55 and 0.73 and again reliability was only fair or poor in this direction. The greater variation in the proximal-distal direction might be related to the shape of the foot, as the foot is longer in this direction compared with the width of the foot. The clinical impact was again evident, PP and PTI values showed a worse reliability than the spatial parameters. The experienced observers (observers 1 and 4 ) had the best intra-observer reliability for multi-masking that may indicate that experience is an important factor.

The SEM values of the pressure characteristics are, contrarily to those of the coordinates, directly applicable in order to evaluate 
the clinical context in which this masking technique could be used. If the reported SEM values found for the multi-masking procedure (Table 3) are considered in view of the minimum PP values, it can be observed that this represent a load of $52 \%$ for the first MTH, $23.7 \%$ for the second $\mathrm{MTH}, 10 \%$ for the third MTH, $28.75 \%$ for the fourth MTH and $56.4 \%$ for the fifth MTH. Contrarily, if the maximum PP values of this study are considered the SEM values represent only a relative load ranging from 3.4\% until 6.5\%. From these results it can be observed that for some patients a considerable pressure alteration is needed before a therapeutic intervention can be adequately tracked.

The use of an X-ray image during the masking procedure is not a commonly used approach in plantar pressure related research. Xray views were only used to inform the observer about the morphology of the respective foot, as none of the observers examined the subjects' feet clinically. We believe that the use of these X-rays did not affect the repeatability of the masking. However, to investigate this study with a different design is necessary, and is beyond the scope of this investigation.

The resolution of the pressure platform is a key element in this investigation and needs special consideration because it may influence the outcome. A higher resolution (number of sensors/ $\mathrm{cm}^{2}$ ) has a tendency to bias to a higher variation, especially when small masks are used. A possible explanation for the results may therefore be related to the high resolution of the plantar pressure platform used in this study.

Only De Cock et al. [18] studied the repeatability of masking previously. However, their study did not investigate the repeatability of masking itself but only the repeatability of the related temporal parameters after positioning of small masks in a healthy population. They concluded that it was a reliable method to investigate temporal pressure characteristics.

In gait analysis research, data are often pooled across three or five steps in order to produce mean values (ensemble averages); and represent an international accepted method to partially overcome the step-to-step variation of human gait [24]. We further explored the value of ensemble averaging to increase the robustness of the data analysis concerning inter-observer agreement. Results showed that a variation $<10 \%$ was found between the ensemble averages of the MTH, which represents a reasonable gross level of agreement. However, this method is time consuming and therefore it can be questioned if it can be easily implemented in clinical practice.

Several limitations of this study are evident. First, the method using a coordinate system to measure the position of the masks has disadvantages, as the mask was represented by one single point, being at its centre. This needs in depth consideration with respect to the algorithms of the pressure platform software, as once masking is done and validated by the clinician, pressure characteristics are determined for the four involved sensors. This implies that it may be possible that a variation of $2 \mathrm{~mm}$ does not change the PP and PTI value and in other cases it may have a tremendous impact. Therefore, we did not only investigate the reliability of PP and PTI values using small masks, because nothing could then be concluded on the accuracy of masking itself.

Second, the reliability was only tested in a hallux valgus population. Future investigations including a variety of foot pathologies should provide additional benchmark data, to fully appreciate the scientific value of the manual masking technique as a tool to report on a variety of clinical applications.

\section{Conclusion}

Using a method based upon 2D coordinates the inter- and intraobserver reliability of masking within plantar pressure measurements was reported. Taken together, manual small masking in order to define pressure characteristics in the forefoot can be seriously questioned. The use of this method may generate important variability which makes it unsuitable for clinical use, despite the fact of being flexible and easy.

\section{Acknowledgments}

We are indebted to Barbara Callewaert, Ilse Heyvaert and Annemie Moerloos for their participation as observer to this study. Jos Nijs is acknowledged for his statistical advice.

\section{Conflict of interest}

The authors have no financial and personal relationships with other people or organizations that could inappropriately influence (bias) their work.

\section{References}

[1] Santos D, Carline T, Richmond R, Abboud RJ. A review of the effects of external pressures on skin blood flow. Foot 2003;13:185-9.

[2] Schmiegel A, Rosenbaum D, Schorat A, Hilker A, Gaubits M. Assessment of foot impairment in rheumatoid arthritis patients by dynamic pedobarography. Gait Posture 2007;27(1):110-4.

[3] Lavery LA, Armstrong DG, Vela SA, Quebedeaux TL, Fleischli SG. Practical criteria for screening patients at high risk for diabetic foot ulceration. Arch Intern Med 1998;158:157-62.

[4] Lüninghake F, Tillman K, Russlies M. Biomechanical changes following surgical treatment of MTP-1-Osteoarthritis. Foot 2002;12:171-81.

[5] Femery V, Moretto P, Renaut H, Thévenon A. Measurement of plantar pressure distribution in hemiplegic children: changes to adaptive gait patterns in accordance with deficiency. Clin Biomech 2002;17:406-13.

[6] Lott DJ, Hastings MK, Commean PK, Smith KE, Mueller MJ. Effect of footwear and orthotic devices on stress reduction and soft tissue strain of the neuropathic foot. Clin Biomech 2007;22:353-9.

[7] Van Gheluwe B, Dananberg HJ, Hagman F, Vanstaen K. Effects of hallux limitus on plantar foot pressure and foot kinematics during walking. J Am Podiatr Med Assoc 2006;96(5):428-35.

[8] Ringleb SI, Kavros SJ, Kotajarvi BR, Hansen DK, Kitaoka HB, Kaufman KR. Changes in gait associated with acute stage II posterior tibial tendon dysfunction. Gait Posture 2006;25:555-64.

[9] Woodburn J, Helliwell P. Observations on the F-Scan in-shoe pressure measuring system. Clin Biomech 1996;11(5):301-4.

[10] Boyd LA, Bontrager EL, Mulroy SJ, Perry JP. The reliability and validity of the Novel Pedar System of in-shoe pressure measurement during free ambulation. Gait Posture 1997;5:165.

[11] Chesnin KJ, Selby-Silverstein L, Besser MP. Comparison of an in-shoe pressure measurement device to a force plate: concurrent validity of center of pressure measurements. Gait Posture 2000;12:128-33.

[12] Nicolopoulos CS, Anderson EG, Solomonidis E, Giannoudis PV. Evaluation of the gait analysis FSCAN pressure system: clinical tool or toy? Foot 2000;10:124-30.

[13] Kernozek TW, LaMott EE. Comparisons of plantar pressures between the elderly and young adults. Gait Posture 1994;3:143-8.

[14] Bryant A, Tinley P, Singer K. Plantar pressure distribution in normal, hallux valgus and hallux limitus feet. Foot 1999;9:115-9.

[15] Bus SA, Maas M, de Lange A, Michels RPJ, Levi M. Elevated plantar pressures in neuropathic diabetic patients with claw/hammer toe deformity. J Biomech 2004;19(6):629-38.

[16] Cavanagh PR, Rodgers MM, Liboshi A. Pressure distribution in symptom-free feet during barefoot standing. Foot Ankle 1987;7(5):262-75.

[17] Widhe T. Foot deformities at birth: a longitudinal prospective study over a 16 year period. J Pediatr Orthop 1997;17:20-4.

[18] De Cock A, De Clercq D, Willems T, Witvrouw E. Temporal characteristics of foot roll-over during barefoot jogging: reference data for young adults. Gait Posture 2005;21(4):432-9.

[19] De Cock A, Willems T, Witvrouw E, Vanrenterghem J, De Clercq D. A functional foot type classification with cluster analysis based on plantar pressure distribution during jogging. Gait Posture 2006;23:339-47.

[20] Willems T, De Clercq D, Delbaere K, Vanderstraeten G, De Cock A, Witvrouw E. A prospective study of gait related risk factors for exercised-related lower leg pain. Gait Posture 2006;23:91-8.

[21] Van Gheluwe B, Dananberg HJ. Changes in plantar foot pressure with in-shoe varus or valgus wedging. J Am Podiatr Med Assoc 2004;94(1):1-11.

[22] Harrison AJ, Folland JP. Investigation of gait protocols for plantar pressure measurements of non-pathological subjects using a dynamic pedobarograph. Gait Posture 1997;6:50-5.

[23] Youdas JW, Carey JR, Garett TR. Reliability of measurements of cervical spine range of motion-comparison of three methods. Phys Ther 1991:71:98-106.

[24] Hughes J, Phil M, Clark P, Linge K, Klenerman L. A comparison of 2 studies of the pressure distribution under the feet of normal subjects using different equipment. Foot Ankle Int 1993;14:514-9. 